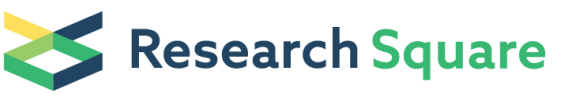 \\ Preprints are preliminary reports that have not undergone peer review. \\ They should not be considered conclusive, used to inform clinical practice, or referenced by the media as validated information.
}

\section{Development and Characterization of SSR Markers in the Gossypium Barbadense Genome}

\section{Wenjuan ZHONG}

Sichuan Academy of Agricultural Sciences, Industrial Crop Research Institute https://orcid.org/00000002-9804-8197

\section{Can YUAN}

Sichuan Academy of Agricultural Sciences, Industrial Crop Research Institute

\section{Zhengjie CHEN}

Sichuan Academy of Agricultural Sciences, Industrial Crop Research Institute

\section{Yonghang ZHOU}

Sichuan Academy of Agricultural Sciences, Industrial Crop Research Institute

\section{Siwei Chen}

Sichuan Academy of Agricultural Sciences, Industrial Crop Research Institute

\section{Qingxia TANG}

Sichuan Academy of Agricultural Sciences,Industrial Crop Research Institute

\section{Chao ZHANG}

Sichuan Academy of Agricultural Sciences, Industrial Crop Research Institute

\section{Yiyun GONG}

Sichuan Academy of Agricultural Sciences, Industrial Crop Research Institute

\section{Zehu YANG}

Sichuan Academy of Agricultural Sciences, Industrial Crop Research Institute

\section{Zhengxuan MAO}

Sichuan Academy of Agricultural Sciences, Indutrial Crop Research Institute

\section{Fangsheng MU ( $\nabla$ fshmu@163.com)}

Sichuan Academy of Agricultural Sciences, Institute Crop Research Institute https://orcid.org/00000001-8671-7974

\section{Peicheng Jl}

Sichuan Academy of Agricultural Sciences, Institute Crop Research Institute

\section{Research}

Keywords: G. barbadense, Simple sequence repeats (SSRs), Microsatellite markers, Genetic diversity analysis

Posted Date: September 14th, 2021 
DOI: https://doi.org/10.21203/rs.3.rs-870780/v1

License: (c) (1) This work is licensed under a Creative Commons Attribution 4.0 International License. Read Full License 


\section{Abstract}

\section{Background}

The fiber quality and resistance traits of Gossypium barbadense are considerably better than that of other Gossypium species. Simple sequence repeats (SSRs) are user friendly, low cost markers widely used in genetic studies. However, most SSRs have been developed from G. hirsutum, G. arboreum, and $G$. raimondii; no genome-wide SSRs have been developed from $G$. barbadense.

The de novo sequences of $G$. barbadense cv. Xinhai21 were utilized to develop SSR markers and scanned to detect SSRs using the MlcroSAtellite (http://pgrc.ipk-gaters/eben.de/misa/) identification tool. And then in silico PCR analysis was conducted to evaluate these primers polymorphism in five Gossypium species.

Results

In total, 85,582 SSRs were identified with different motifs. 153,560 primer pairs were successfully designed for 73,419 SSRs. In silico analysis, we found that 8,466 primer pairs of 3,288 SSRs yielded one product (monomorphic) simultaneously in five Gossypium species. two Gossypium species (30 G. hirsutum and $27 \mathrm{G}$. barbadense accessions) were successfully separated by 300 primer pairs with the polymorphism information content (PIC) ranging from 0.00 to 0.93 .

Conclusion

These newly developed SSR markers will be helpful for the construction of genetic linkage maps, genetic diversity analyses, QTL mapping, and molecular breeding of Gossypium species.

\section{Background}

Cotton (Gossypium spp.) is one of the most popular sources of natural fiber and oil. The Gossypium genus contains 45 diploid $(2 n=26)$ and 6 tetraploid $(2 n=52)$ species (Hawkins et al. 2006; Grover et al. 2015). Only four Gossypium species, including two tetraploids and two diploids, produce spinnable fiber. Gossypium barbadense (also known as sea-island cotton, extra-long staple cotton, American Pima, and Egyptian cotton) is one of two allotetraploid cultivated cotton species that produces extra-long fibers used in the manufacturing of superior textiles and contributes to $8 \%$ of the world's cotton output (Zhang et al. 2008). Due to its excellent fiber quality and high resistance to verticillium wilt, $G$. barbadense is considered an ideal genetic donor for improving the characteristics of $G$. hirsutum such as fiber quality and disease resistance. However, lack of genome-wide molecular markers in G. barbadense for genetic research and marker-assisted selection (MAS).

Simple sequence repeats (SSRs), or microsatellites, are repeats of 1-6 bp nucleotides representing high frequency, distribution, co-dominance, reproducibility, and high polymorphism (Powell et al. 1996; Gupta and Varshney 2000). Because of its aforementioned advantages, SSR markers have been widely used in 
molecular finger-printing, genetic diversity analysis, mapping genetic linkage, and marker-assisted selection in many species over the past decades (Reddy et al. 2001; Feng et al. 2016). In addition, SSRs developed from expressed sequence tags (EST) possess a higher conserved sequence; hence the transferability of EST-SSRs is higher than that of genomic-SSRs across related species (Zhou et al. 2016). Therefore, EST-SSRs are appropriate for integrating different genetic linkage maps, QTL mapping, and evolutionary correlation (Tani et al. 2003).

Numbers of genomic SSRs have been developed in Gossypium species such as G. hirsutum, G. arboreum, and $G$. raimondii, and are widely used for constructing genetic linkage maps. In the absence of a reference genome for cotton, Reddy et al obtained more than 10,000 microsatellite-containing fragments in G. hirsutum, as well as designed primers for 307 out of 588 SSR markers, of which, $49 \%$ showed length polymorphism by polymerase chain reaction (PCR) amplification (Reddy et al. 2001). In the G. hirsutum cv. Guazuncho 2 cDNA library, 846 clones were sequenced, but only 392 sequences containing SSRs had previously designed primers (Nguyen et al. 2004). Similarly, 966 sequences containing SSRs were identified from fiber/ovule cDNA libraries constructed for $G$. hirsutum, from which, 489 SSR primer pairs were developed (Han et al. 2006). Based on the ESTs derived from G. arboreum fibers 7-10 days postanthesis, 931 ESTs contained SSRs, of which, 544 had previously designed primers; only 99 were polymorphic between G. hirsutum cv. TM-1 and G. barbadense cv. Hai7124 (Han et al. 2004). Subsequently, genome of Gossypium species was sequenced and 136,345 SSRs were identified from $775.2 \mathrm{Mb}$ sequences in G. raimondii; 112,177 primer pairs were designed for these SSRs (Zou et al. 2012). Moreover, $100,290,83,160$, and 56,937 SSRs were developed from the sequences of G. hirsutum, G. arboreum, and G. raimondii, respectively; thousands of primer pairs were designed (Wang et al. 2015). A number of SSR markers can be available from the CottonGen database (www.cottongen.org), however, almost all were derived from G. hirsutum (Zhang et al. 2005; Qayyum et al. 2009; Wang et al. 2015), G. abroreum (Guo et al. 2006; Liu et al. 2006; Kantartzi et al. 2009), or G. raimondii (Zou et al. 2012), while only a few SSRs were designed based on $G$. barbadense sequences, including 214 sequences obtained from G. barbadense cv. acc3-79 (Zhang et al. 2009).

G. barbadense cv. Xinhai21 produces extra-long fibers and is used in the production of superior textiles, its genome sequence had been sequenced and gene annotation had been studied in 2015 (Liu et al. 2015). In this study, the de novo sequences of $G$. barbadense cv. Xinhai21 were utilized to develop genomic SSR markers and primer pairs were designed for the SSR markers. Further, in silico analysis were performed to evaluate the polymorphism of the SSR primer pairs in five Gossypium species. To evaluate the potential usefulness of the SSR markers, genetic diversity analysis was conducted in different $G$. hirsutum and $G$. barbadense species. Our results will help improve the application of SSR markers and uncover the genetic basis of dominant $G$. barbadense traits.

\section{Results}

Characteristics of genome-wide SSRs in G. barbadense cv. Xinhai21 
The published $\sim 2264 \mathrm{Mb}$ of G. barbadense cv. Xinhai21 genome sequence was utilized to develop SSRs with different types of desirable repeat motifs ranging from mono- to hexa-nucleotides. As a result, a total of 85,582 SSRs were identified genome-wide. Among them, 78,109 SSRs were physically mapped to the 26 chromosomes of $G$. barbadense with an average density of 38.20 per Mb. However, the density of SSRs in the $A_{t}$ sub-genome was 33.50 per $M b$, which was smaller than the $D_{t}$ sub-genome (46.17 per $\mathrm{Mb}$ ) (Tables 1; Table 2; Fig. 1). Chromosome D07 had the highest density (52.36 per Mb), while chromosome A04 had the lowest density (28.27 per Mb) (Table 2). In total, 476 types of repeat motifs were detected for mono- (2), di- (4), tri- (10), tetra- (32), penta- (95), and hexa-nucleotides (333) (Table S1). The distributions of mono- to hexa-nucleotide SSRs in the $A_{t}$ and $D_{t}$ sub-genomes were different. Overall, the numbers of SSRs in $A_{t}$ was greater than in $D_{t}$, even more than two times greater for mono-nucleotide SSRs (Table 3). From the occurrence frequency of different repeat motifs, hexa-nucleotide repeats were the most abundant $(31,843,37.21 \%)$, followed by tri- $(18,200,21.27 \%)$, penta- $(12,827,14.99 \%)$, di- $(12,375$, $14.46 \%)$, tetra- $(7,089,8.28 \%)$, and mono-nucleotides $(3,248,3.80 \%)$ (Table 3$)$. Having ranked the different motifs, the top 10 were rich in AAT/ATT $(12,870,13.14 \%)$, AAAAAT/ATTTTT $(9,616,9.82 \%)$, AT/AT $(8,218$, 8.39\%), AAAAT/ATTTT (5,188, 5.30\%), A/T (4,690, 4.79\%), AAAT/ATTT (4,074, 4.16\%), AAG/CTT (4,015, 4.10\%), AAAAAG/CTTTTT (3,609, 3.69\%), AG/CT (3,272, 3.34\%), and AATCAG/ATTCTG $(2,366,2.42 \%)$ (Table 4). Similarly, the numbers of the top 10 motifs in $A_{t}$ were greater than in $D_{t}$. Additionally, some motifs were more frequent in $A_{t}$ than in $D_{t}$. For instance, the number of the AATCAG motif was 2,324 in $A_{t}$, but 42 in $D_{t}$, and the number of the ACAGG motif was 211 in $A_{t}$, but 1 in $D_{t}$ (Table S2).

Table 1

Overall frequency of SSRs in the G. barbadense cv. Xinhai21

\begin{tabular}{|llll|}
\hline Genome & number of SSRs & Genome Size $(\mathrm{Mb})$ & SSR/Mb \\
\hline Sub genome $A_{t}$ & 47,663 & 1442.7 & 33.04 \\
\hline Sub genome $D_{t}$ & 37,919 & 820.8 & 46.20 \\
\hline Whole genome & 85,582 & 2263.5 & 37.81 \\
\hline
\end{tabular}


Table 2

Distribution and average density of SSRs and SSR marker primer sets mapped on G. barbadense cv. Xinhai21 chromosomes

\begin{tabular}{|c|c|c|c|c|c|}
\hline Chromosome & Analysis size (Mb) & Total_SSRs & SSR/Mb & SSRs_with_primers & SSR/Mb \\
\hline$A_{t} 01$ & 63.84 & 2,367 & 37.08 & 1,900 & 29.76 \\
\hline$A_{t} 02$ & 98.77 & 2,837 & 28.72 & 2,352 & 23.81 \\
\hline $\mathrm{A}_{t} \mathrm{O3}$ & 104.2 & 3,202 & 30.73 & 2,608 & 25.03 \\
\hline $\mathrm{A}_{\mathrm{t}} \mathrm{O4}$ & 80.37 & 2,272 & 28.27 & 1,794 & 22.32 \\
\hline$A_{t} 05$ & 100.73 & 4,272 & 42.41 & 3,414 & 33.89 \\
\hline $\mathrm{A}_{\mathrm{t}} \mathrm{O6}$ & 115.58 & 3,331 & 28.82 & 2,731 & 23.63 \\
\hline $\mathrm{A}_{t} 07$ & 93.49 & 3,354 & 35.88 & 2,686 & 28.73 \\
\hline$A_{t} 08$ & 116.27 & 3,650 & 31.39 & 2,970 & 25.54 \\
\hline$A_{t} 09$ & 76.81 & 2,979 & 38.78 & 2,353 & 30.63 \\
\hline$A_{t} 10$ & 110.16 & 3,758 & 34.11 & 2,956 & 26.83 \\
\hline$A_{t} 11$ & 115.13 & 4,125 & 35.83 & 3,331 & 28.93 \\
\hline$A_{t} 12$ & 103.35 & 3,636 & 35.18 & 2,940 & 28.45 \\
\hline$A_{t} 13$ & 108.51 & 3,343 & 30.81 & 2,707 & 24.95 \\
\hline$A_{t}$ & 1287.21 & 43,126 & 33.5 & 34,742 & 26.99 \\
\hline$D_{t} 01$ & 60.1 & 2,662 & 44.29 & 2,217 & 36.89 \\
\hline$D_{t} 02$ & 66.13 & 2,765 & 41.81 & 1,787 & 27.02 \\
\hline$D_{t} 03$ & 50.69 & 2,167 & 42.75 & 1,787 & 35.26 \\
\hline$D_{t} 04$ & 49.32 & 2,151 & 43.61 & 1,788 & 36.26 \\
\hline Dt05 & 59.32 & 3,076 & 51.85 & 2,559 & 43.14 \\
\hline$D_{t} 06$ & 60.1 & 2,650 & 44.09 & 2,193 & 36.49 \\
\hline$D_{t} 07$ & 55.25 & 2,893 & 52.36 & 2,363 & 42.77 \\
\hline$D_{t} 08$ & 63.71 & 2,977 & 46.73 & 2,433 & 38.19 \\
\hline$D_{t} 09$ & 47.68 & 2,287 & 47.97 & 1,887 & 39.58 \\
\hline
\end{tabular}




\begin{tabular}{|llllll|}
\hline Chromosome & Analysis size $(\mathrm{Mb})$ & Total_SSRs & SSR/Mb & SSRs_with_primers & SSR/Mb \\
\hline $\mathrm{D}_{\mathrm{t}} 10$ & 62.34 & 2,800 & 44.91 & 2,279 & 36.56 \\
$\mathrm{D}_{\mathrm{t}} 11$ & 65.08 & 3,055 & 46.94 & 2,512 & 38.6 \\
$\mathrm{D}_{\mathrm{t}} 12$ & 58.26 & 2,782 & 47.75 & 2,325 & 39.91 \\
$\mathrm{D}_{\mathrm{t}} 13$ & 59.7 & 2,718 & 45.53 & 2,227 & 37.3 \\
$\mathrm{D}_{\mathrm{t}}$ & 757.67 & 34,983 & 46.17 & 28,357 & 37.43 \\
\hline Total & 2044.88 & 78,109 & 38.2 & 63,099 & 30.86 \\
\hline
\end{tabular}

Table 3

Distribution of six types of SSR in the G. barbadense cv. Xinhai21 genome

\begin{tabular}{|lllll|}
\hline Repeat Motifs & Sub genome $\mathbf{A}_{\mathbf{t}}$ & Sub genome $\boldsymbol{D}_{\mathbf{t}}$ & Whole genome & Ration (\%) \\
\hline Mono-nucleotide & 2,259 & 989 & 3,248 & 3.80 \\
\hline Di-nucleotide & 6,767 & 5,608 & 12,375 & 14.46 \\
\hline Tri-nucleotide & 10,133 & 8,067 & 18,200 & 21.27 \\
\hline Tetra-nucleotide & 3,554 & 3,535 & 7,089 & 8.28 \\
\hline Penta-nucleotide & 7,375 & 5,452 & 12,827 & 14.99 \\
\hline Hexa-nucleotide & 17,575 & 14,268 & 31,843 & 37.21 \\
\hline
\end{tabular}


Table 4

Top 10 repeat motifs in sub genome At, Dt and whole genome of $G$. barbadense cv. Xinhai21

\begin{tabular}{|c|c|c|c|c|c|c|c|c|}
\hline \multicolumn{3}{|c|}{ Whole genome } & \multicolumn{3}{|c|}{ Sub genome $A_{t}$} & \multicolumn{3}{|c|}{ Sub genome $D_{t}$} \\
\hline Motifs & Total & $\begin{array}{l}\text { Ratio } \\
(\%)\end{array}$ & Motifs & Total & $\begin{array}{l}\text { Ratio } \\
(\%)\end{array}$ & Motifs & Total & $\begin{array}{l}\text { Ratio } \\
(\%)\end{array}$ \\
\hline AAT/ATT & 12870 & 13.14 & AAT/ATT & 7738 & 16.76 & AAT/ATT & 5132 & 13.93 \\
\hline AAAAAT/ & \multirow[t]{2}{*}{9616} & \multirow[t]{2}{*}{9.82} & AAAAAT/ & \multirow[t]{2}{*}{5560} & \multirow[t]{2}{*}{12.04} & AAAAAT/ & \multirow[t]{2}{*}{4056} & \multirow[t]{2}{*}{11.01} \\
\hline АTTTTT & & & ATTTTT & & & АTTTTT & & \\
\hline AT/AT & 8218 & 8.39 & AT/AT & 4584 & 9.93 & AT/AT & 3634 & 9.86 \\
\hline AAAAT/ & \multirow[t]{2}{*}{5188} & \multirow[t]{2}{*}{5.30} & AAAAT/ & \multirow[t]{2}{*}{3326} & \multirow[t]{2}{*}{7.20} & \multirow[t]{2}{*}{ AAAT/ATTT } & \multirow[t]{2}{*}{1992} & \multirow[t]{2}{*}{5.41} \\
\hline ATTTT & & & ATTTT & & & & & \\
\hline $\mathrm{A} / \mathrm{T}$ & 4690 & 4.79 & $\mathrm{~A} / \mathrm{T}$ & 3252 & 7.04 & AAG/CTT & 1966 & 5.34 \\
\hline \multirow[t]{2}{*}{ AAAT/ATTT } & \multirow[t]{2}{*}{4074} & \multirow[t]{2}{*}{4.16} & AATCAG/ & \multirow[t]{2}{*}{2324} & \multirow[t]{2}{*}{5.03} & AAAAT/ & \multirow[t]{2}{*}{1862} & \multirow[t]{2}{*}{5.05} \\
\hline & & & ATTCTG & & & ATTTT & & \\
\hline AAG/CTT & 4015 & 4.10 & AAAT/ATTT & 2082 & 4.51 & AG/CT & 1600 & 4.34 \\
\hline AAAAAG/ & \multirow[t]{2}{*}{3609} & \multirow[t]{2}{*}{3.69} & \multirow[t]{2}{*}{ AAG/CTT } & \multirow[t]{2}{*}{2049} & \multirow[t]{2}{*}{4.44} & AAAAAG/ & \multirow[t]{2}{*}{1594} & \multirow[t]{2}{*}{4.33} \\
\hline стTTTT & & & & & & сTTTTT & & \\
\hline \multirow[t]{2}{*}{$\mathrm{AG} / \mathrm{CT}$} & \multirow[t]{2}{*}{3272} & \multirow[t]{2}{*}{3.34} & AAAAAG/ & \multirow[t]{2}{*}{2015} & \multirow[t]{2}{*}{4.36} & \multirow[t]{2}{*}{$\mathrm{A} / \mathrm{T}$} & \multirow[t]{2}{*}{1438} & 3.90 \\
\hline & & & СTTTTT & & & & & \\
\hline
\end{tabular}

\section{Development of Genome-wide SSR primers}

A total of $69,750(81.50 \%)$ out of 85,582 SSRs were successfully applied to design primer pairs from their unique flanking sequences, which obtained 209,250 primer pairs (Table S3). The majority of designed SSR primer sets were hexa-nucleotides $(26,867,38.52 \%)$, followed by tri- $(13,194,18.92 \%)$, penta- $(11,072$, $15.87 \%)$, di- $(10,146,14.55 \%)$, tetra- $(5,774,8.27 \%)$, and mono-nucleotides $(2,697,3.86 \%)$ (Table S3). Moreover, of these 69,750 designed SSR primers, 63,099 SSRs anchored to the 26 chromosomes of $G$. barbadense cv. Xinhai21 with an average marker density of 30.86 SSR markers per Mb (Table 2).

Although the number of SSRs in $A_{t}$ was greater than in $D_{t}$, the average marker density in $D_{t}(37.43$ per $\mathrm{Mb})$ was greater than in $A_{t}(26.99$ per $\mathrm{Mb})$ since the size of $A_{t}(\sim 1,442.7 \mathrm{Mb})$ was much larger than $D_{t}(\sim$ $820.8 \mathrm{Mb}$ ). Chromosome D05 had the highest density (43.14 per Mb), whereas chromosome A04 had the lowest density (22.32 per Mb) (Table 2).

In silico PCR analysis 
The polymorphism of SSR marker primer pairs were evaluated by an in silico PCR analysis. It was revealed that the PCR products of 209,250 primer pairs varied with several PCR products including 0, 1, 2, 3 , or $>3$ products in $G$. barbadense $c v$. Xinhai21. Of these primers, 153,560 primer pairs were retained because they produced 1, 2, or 3 products. Specifically, 89,940 primer pairs produced 1 product, 49,462 produced 2 products, and 14,158 produced 3 products (Table 5). Thus, these three types of primer pairs were used to evaluate polymorphism in the other four Gossypium species. Among the four Gossypium species, the number of primer pairs that produced 0 products ranged from $23,378-73,107$, primers that produced 1 product ranged from 42,145-81,590, primers that produced 2 products ranged from 4,89744,602 , primers that produced 3 products ranged from 1,405-18,827, and primers that produced $>3$ products ranged from $724-24,608$ (Table 5). Additionally, it is important to note that 8,466 primer pairs involving 3,288 SSR markers yielded 1 product in all five Gossypium species (Table S4).

Table 5

Number of products of primer sets designed from G. barbadense cv. Xinhai21 generated through in silico analysis in G. barbadense cv. acc3-79, G. arboretum, G. hirsutum and G. raimondii genomes

\begin{tabular}{|llllll|}
\hline Genome & $\mathbf{0}$ product & 1 product & 2 products & 3 products & > 3 products \\
\hline G. barbadense cv. & 4,306 & 89,940 & 49,462 & 14,158 & 51,384 \\
Xinhai21 & $(2.1 \%)$ & $(43.0 \%)$ & $(23.6 \%)$ & $(6.8 \%)$ & $(24.5 \%)$ \\
\hline G. barbadense cv.acc & 23,378 & 42,145 & 44,602 & 18,827 & 24,608 \\
3-79 & $(15.2 \%)$ & $(27.4 \%)$ & $(29.0 \%)$ & $(12.3 \%)$ & $(16.0 \%)$ \\
G. arboreum (BGI) & 63,383 & 81,590 & 5,775 & 1,568 & $1,244(0.8 \%)$ \\
& $(41.3 \%)$ & $(53.1 \%)$ & $(3.8 \%)$ & $(1.0 \%)$ & \\
G. hirsutum $(\mathrm{TM}-1)$ & 28,044 & 75,647 & 41,701 & 5,842 & $2,326(1.5 \%)$ \\
& $(18.3 \%)$ & $(49.3 \%)$ & $(27.2 \%)$ & $(3.8 \%)$ & \\
\hline G. raimondii (JGI) & 73,107 & 73,427 & 4,897 & 1,405 & $724(0.5 \%)$ \\
& $(47.6 \%)$ & $(47.8 \%)$ & $(3.2 \%)$ & $(0.9 \%)$ & \\
\hline
\end{tabular}

\section{PCR amplification and genetic diversity analysis}

Three hundred SSR marker primer pairs (Table S7) were randomly selected from the 8,466 primer pairs, which were used for PCR amplification in the $30 \mathrm{G}$. hirsutum and $27 \mathrm{G}$. barbadense accessions (Table S8). Of these 300 primer pairs, 139 (46.33\%) successfully amplified clear, single, expect fragments with noticeable differnces among the 57 Gossypium accessions, while 94 showed no difference, 57 showed no expect amplification, and 10 amplified nothing (Fig. 2). Therefore, the genotype bands of the 139 primer pairs were selected for PIC calculation and cluster analysis. Ultimately, PIC values were $0.00-0.56$ with an average of 0.05 in the $30 \mathrm{G}$. hirsutum accessions and PIC values varied from 0.00 to 0.93 with a mean 0.07 in the $27 \mathrm{G}$. barbadense accessions (Table S7). In the case of a similarity coefficient $\geq 0.61$, the 57 Gossypium accessions were divided into two subgroups. One group contained the $30 \mathrm{G}$. hirsutum accessions and the other contained the 27 G. barbadense accessions (Fig. 3), confirming that these markers developed from $G$. barbadense cv. Xinhai21 could be used to determine genetic diversity in cotton and differentiate G. barbadense and G. hirsutum. 


\section{Analysis of GO terms and KEGG pathways for genes containing SSR markers}

Genes of G. barbadense cv. Xinhai21 were blasted against the genome sequence of G. barbadense cv. acc3-79, which resulted in obtaining 74,371 genes. Of these genes, 50,713 had GO ID information, 20,539 had KEGG ontology, and 13,952 genes had a pathway ID (Table S5). There were 9,378 genes containing at least one SSR marker. Among them, 6,738 genes had GO ID information, 3,014 had KEGG ontology, and 2,002 had a pathway ID. The number of genes on each chromosome ranged from $204\left(A_{t} 04\right)$ to 533 $\left(A_{t} 05\right)$ (Table S6). Moreover, the GO enrichment of genes containing SSR markers were involved in many metabolic processes, such as zinc binding, ubiquitin-protein transferase activity, transcription factor activity, sucrose metabolism, polysaccharide catabolism, hydrolase activity, and microtubule-associated complex. As for the KEGG pathways, processes included ubiquitin-mediated proteolysis, plant hormone signal transduction, starch and sucrose metabolism, and vasopressin-mediated water reabsorption (Table S6).

\section{Discussion}

Comparison of SSR markers ddeveloped in the present study and previous studies

Although the development of genome-wide SSR markers in G. hirsutum, G. arboreum, and G. raimondii were reported previously (Wang et al. 2015; Zou et al. 2012), there are no reports for G. barbadense. In this study, using the de novo sequences of G. barbadense cv. Xinhai21 allowed for the identification of 85,582 SSR markers with an average density of 37.81 per Mb. The number of SSRs identified in this study is greater than those identified by Wang et al. for $G$. arboreum $(83,160)$ and $G$. raimondii $(56,937)$, but less those identified by Zou et al. for $G$. raimondii $(136,345)$ and Wang et al. for $G$. hirsutum $(100,290)$. The density of SSRs in G. barbadense cv. Xinhai21 was close to $G$. hirsutum (41.2 per Mb), but much lower than G. arboreum (49.1 per Mb) and G. raimondii (74.8 per Mb and 175.88 per Mb) (Wang et al. 2015; Zou et al. 2012). Interestingly, the distributions of microsatellite length in this study were similar to those identified by Wang et al. both ranking as hexa- (37.21\% vs. $39.40 \%)$, tri- ( $21.27 \%$ vs. $22.40 \%)$, penta( $14.99 \%$ vs. $14.90 \%)$, di- (14.46\% vs. $12.40 \%)$, tetra- (8.28\% vs. $9.00 \%)$, and mono-nucleotides (3.80\% vs. $1.80 \%$ ) (Wang et al. 2015). Additionally, the AAT/ATT motif was the most abundant in G. barbadense cv. Xinhai21, accounting for $13.14 \%$, which was consistent with the findings for G. hirsutum $(14.04 \%)$. Although the AAT/ATT motif was the second degree abundant, it accounted for 11.15 and $12.69 \%$ in $G$. raimondii and G. arboreum, respectively (Wang et al. 2015). These results suggest that the pattern of microsatellite distribution is relatively conserved in Gossypium species and remains unchanged in the formation of allotetraploid cotton.

\section{Genotyping advantage of monomorphic SSR markers}

Only one PCR product yielded in PCR amplification using monomorphic SSR markers. Consequently, it is easy to distinguish and analyze the genotype. Using 17 monomorphic SSR markers, 14 were identified 
that showed differences in alleles among 21 individuals, indicating that there was considerable variation among the flanking sequences of monomorphic SSR markers (Nazareno and Reis 2011). In this study, 8,466 primer pairs yielded one product in in silico PCR analysis, of which, 300 monomorphic SSR marker primer pairs were used for PCR amplification in the G. hirsutum and G. barbadense accessions; 139 $(46.33 \%)$ out of 300 amplified clear, single fragments, which was agreed with the results of the in silico PCR analysis, and successfully classified these two Gossypium species. Additionally, the effective amplification of the primer sets $(46.33 \%)$ was close to that $(50.25 \%$; 266 out of 511$)$ identified by Wang et al. (2015), suggesting that monomorphic SSR markers can be widely used in genetic and evolutionary studies.

\section{Application of SSRs developed from G. barbadense in cotton breeding}

The quality of G.bardadense fiber is very good, thus one breeding goal is to introduce good fiber alleles into other high yield Gossypium species, in order to breed better quality fiber and higher yield varieties. According to the $\mathrm{GO}$ enrichment analysis, many genes containing SSRs were found to be possibly involved in fiber development (Table S6). For instance, 11 SSRs located on chromosomes A05 (1), A12 (2), D04 (1), D08 (3) and D12 (4) were identified with the term G0: 0009733, which is related to auxin biosynthesis and plant hormone signal transduction.

Expression of the IAA biosynthetic gene, iaaM, by transgenic methods increased the IAA levels during the fiber initiation stage, resulting in significantly improved lint yield and fiber fineness (Zhang et al. 2011). Some studies inferred that secondary metabolic compounds, such as phenylpropanoids, could affect cotton fiber (Hovav et al. 2008; Yang et al. 2006), and phenylpropanoid-related genes exhibited spatial distribution changes during the development of fiber (Yves et al. 2009). There were 25 SSRs associated with phenylpropanoid biosynthesis, which were located on chromosomes A03 (2), A04 (1), A05 (2), A09 (2), A11 (2), A12 (1), A13 (1), D02 (1), D03 (1), D04 (1), D05 (7), D09 (2), D12 (1), D13 (1), and UKA (1), and all are involved in a single pathway, ko00940, that encodes different enzymes, including 0 methyltransferases, peroxidases, transferases, and metabolic enzymes.

As is previously reported, cellulose accounts for more than $94 \%$ of mature cotton fiber (Haigler et al. 2001). Five SSRs identified with the term GO: 0030244 were associated with cellulose biosynthesis and were distributed on chromosomes A02 (2), A06 (1), D05 (1), and D11 (1). The formation of cellulose needs sucrose synthase (Sus) to catalyze uridine diphosphate glucose (UDP-G), the precursor for cellulose (Amor et al. 1995; Ruan 2007). Eight SSRs identified with the term GO: 0016157 were associated with sucrose synthase, regulating starch, and sucrose metabolism (ko00500). Moreover, extensive arrays of microtubules are essential for the elongation of oriented cellulose microfibrils (He et al. 2008). Several $\beta$-tubulin (TUB) genes are highly expressed in elongating cotton fiber cells. Eight genes rescued the lethality phenotype when 9 TUB genes were transformed into the tub2 mutant, which is deficient in b-tubulin (He et al. 2008). Two SSRs located on chromosomes A04 and D05 that were identified with the term GO: 0048487 are related to $\beta$-tubulin binding. Collectively, the SSR markers developed in this study can be used to uncover the genetic basis of fiber development and improve the 
quality of fiber by MAS. Additionally, these SSRs will be helpful for studying other traits, including biotic and abiotic resistance.

\section{Conclution}

In summary, a genome-wide development of SSR markers in sea-island cotton (G. barbadense cv. Xinhai21) was performed. We characterized the genomic SSRs and developed the genome-wide SSR

primer from assembled genomic sequence, also evaluated polymorphism in different species. Furthermore, we conducted GO and KEGG enrichment analysis for genes containing SSRs. These results indicate that the newly development SSR markers can serve as a useful tool for genetic analysis and molecular breeding, especially on the construction of genetic linkage maps, genetic diversity analyses, QTL mapping of important traits, such as fiber quality and biotic resistance traits.

\section{Methods}

\section{Acquisition of genome sequences}

Genome sequence of $G$. barbadense cv. Xinhai21 was available from the NCBI database (https://www.ncbi.nlm.nih.gov/search/all/?term=PRJNA251673), which was used to develop SSR markers. Genome sequences of G. barbadense (acc3-79), G. arboreum (BGI), G. hirsutum (TM-1), and G. raimondii (JGI) were downloaded from the CottonGen database (https://www.cottongen.org), which was used for in silico analysis to evaluate the polymorphism of SSR primer pairs.

\section{Simple sequence repeat (SSR) identify and primer design}

Genome sequences of $G$. barbadense cv. Xinhai21 were scanned to detect SSRs using the MlcroSAtellite (http://pgrc.ipk-gatersleben.de/misa/) identification tool with basic motifs ranging from mono- to hexanucleotides (Thiel et al. 2003). The minimum length criteria were defined as 18, 9, 6, 5, 4, and 3 repeat units for mono-, di-, tri-, tetra-, penta-, and hexa-nucleotide motifs, respectively. Primers were designed based on the flanking sequences of SSR loci using Primer 3 v2.2.3 with the following parameters: 18-27 bp primer length, $57^{\circ} \mathrm{C}-63^{\circ} \mathrm{C}$ melting temperature, $30-70 \%$ GC content, and $100-280$ bp product size (Rozen and Skaletsky 2004). For each SSR, three primer pairs were designed; if one sequence of the two primer pairs was identical, the two pairs were merged into one primer pair.

\section{In silico analysis}

In order to evaluate the polymorphism of the aforementioned SSR primer pairs, an in silico PCR analysis was conducted. First, sequences of SSR marker regions were aligned to the genome sequence of $G$. barbadense cv. Xinhai21 using e-PCR-2.3.11 (ftp://ftp.ncbi.nlm.nih.gov/pub/schuler/e-PCR/) with the following default parameters: 2 bp mismatch, 1 bp gap, 50 bp margin, and 50-1000 bp product size (Kirill et al. 2004; Shi et al. 2014; Wang et al. 2015). Only the SSR marker primer pairs that yielded one 
product (monomorphic), two products, and three products were retained for further analyses. Then, these three types of SSR marker primer pairs were subjected to a BLAST analysis against the assembled contigs of G. barbadense (acc3-79), G. arboreum (BGI), G. hirsutum (TM-1), and G. raimondii (JGI) using e-PCR-2.3.11.

\section{Evaluation of potential usefulness for SSR markers through genetic diversity analysis}

To evaluate the potential usefulness of SSR marker primer pairs, a total of 300 primer pairs that yielded one product simultaneously in G. barbadense cv. Xinhai21, G. barbadense (acc3-79), G. arboreum (BGI), G. hirsutum (TM-1), and $G$. raimondii (JGI) were randomly chosen. Then, primer pairs were experimentally PCR amplified in $30 \mathrm{G}$. hirsutum and $27 \mathrm{G}$. barbadense accessions. Genomic DNA of the $30 \mathrm{G}$. hirsutum and $27 \mathrm{G}$. barbadense accessions were extracted from fresh young leaves using an improved cetyltrimethyl ammonium bromide (CTAB) method (Paterson et al. 1993). PCR amplification was performed on a $20.0 \mu \mathrm{L}$ sample consisting of $10.0 \mu \mathrm{L} 2 \times$ Tag Mastermix (Cat.CW0682L), $1.0 \mu \mathrm{L}(10 \mu \mathrm{M})$ forward primer, $1.0 \mu \mathrm{L}(10 \mu \mathrm{M})$ reverse primer, and $3.0 \mu \mathrm{L}(50 \mathrm{ng} / \mu \mathrm{L})$ gDNA template. The PCR cycling conditions were as follows: 2 min at $94^{\circ} \mathrm{C}, 35$ cycles at $94^{\circ} \mathrm{C}$ for $40 \mathrm{~s}, 35 \mathrm{~s}$ at the annealing temperature for each specific primer, and $60 \mathrm{~s}$ at $72^{\circ} \mathrm{C}$ with a $7 \mathrm{~min}$ extension at $72^{\circ} \mathrm{C}$ in the final cycle. PCR products were separated on $8 \%$ denaturing polyacrylamide gel (PAGE) with $1 \times$ TBE buffer running at $180 \mathrm{~V}$ for $45-$ 60 min and visualized by sliver-staining to check the amplification (Santos et al. 1993). Polymorphism information content (PIC) value was used to assess the allelic polymorphism of each SSR marker, which was calculated by using formula as previous proposed by Liu et al (2015). Then, the genetic relatedness of the $30 \mathrm{G}$. hirsutum and $27 \mathrm{G}$. barbadense accessions was analyzed. After SSR band data were standardized, cluster analysis was conducted based on the similarity coefficients and un-weighted pair group method with arithmetic averages (UPGMA) using the NTSYS-pc v2.10 package (Rohlf 1987; Xie et al. 2006).

\section{GO and KEGG enrichment analyses of genes containing SSRs}

In order to investigate the GO and KEGG enrichment of genes containing at least one SSR, GO terms of $G$. barbadense cv. Xinhai21 genes were annotated first, and then KEGG pathway was annotated since $G$. barbadense cv. Xinhai21 genes have no GO and KEGG enrichment information. In brief, sequences of $G$. barbadense $\mathrm{cv}$. Xinhai21 genes were blasted against the genome sequence of $G$. barbadense $\mathrm{cv}$. acc3-79 whic has GO term and KEGG pathway annotation information, such that obtain the GO and KEGG enrichment information of corresponding genes. Then, the aforementioned SSRs were anchored to the $G$. barbadense $\mathrm{cv}$. Xinhai21 genome to determine the psychical position of SSRs and if gene contains SSR marker. Finally, genes containing SSR(s) were screened from the $G$. barbadense cv. Xinhai21 genes, thus obtaining the GO and KEGG enrichment information.

\section{Abbreviations}

SSR Simple sequence repeats 
PCR Polymerase chain reaction

PIC Polymorphism information content

MAS Marker-assisted selection

EST Expressed sequence tags

QTL Quantitative trait loci

CTAB Cetyltrimethyl ammonium bromide

\section{Declarations}

\section{Acknowledgements}

We thank National Medium-term Gene Bank of Cotton in China and National Cotton Germplasm Resources Platform provided the $30 \mathrm{G}$. hirsutum and $27 \mathrm{G}$. barbadense accessions in Table S8. This work was funded by the National Key Technology R\&D Program, the Ministry of Science and Technology (2016FYD0100203 and 2016YFD0101410).

\section{Authors' contributions}

Mu FS, Zhong WJ and Yuan C conceived and designed the experiments; Chen ZJ, Chen SW, Zhou YH, Ji $P C$, Gong $Y Y$ and Yang ZH planted the materials and assisted in experiments, Zhong WJ , Tang QX, Yuan $C$ performed most of the experiments and analyzed the data. Wen juan Zhong and Zhengjie Chen wrote the manuscript. Fangshen Mu and Ji PC revised the manuscript. Chao Zhang supervised the experiment. All authors read and approved the final manuscript.

\section{Ethics approval and consent to participate}

Not applicable.

\section{Consent for publication}

Not applicable.

\section{Competing interests}

The authors declare no conflict of interest.

\section{References}

Amor Y, Haigler $\mathrm{CH}$, Johnosn S, et al. membrane-associated form of sucrose synthase and its potential role in synthesis of cellulose and callose in plants. Proceedings of the National Academy of Sciences of 
the United States of America.1995;92: 9353-7. https://doi.org/10.1073/pnas.92.20.9353.

Feng SG, He RF, Liu JJ, et al.Development of SSR markers and assessment of genetic diversity in medicinal Chrysanthemum morifolium cultivars. Frontiers in Genetics. 2016;7:113.

https://doi.org/10.3389/fgene.2016.00113.

Grover CE, Zhu X, Grupp KK, et al. Molecular confirmation of species status for the allopolyploid cotton species, Gossypium ekmanianum Wittmack. Genet Resour Crop Evol.2015;62: 103-14. https://doi.org/10.1007/s10722-014-0138-x.

Guo WZ, Zhou BL, Yang LM, et al. Genetic diversity of landraces in Gossypium arboreum L. Race sinense assessed with simple sequence repeat markers. Journal of Intergrative Plant Biology. 2006;48: 1008-1017. https://doi.org/10.1111/j.1744-7909.2006.00316.x.

Gupta PK, Varsheny RK.The development and use of microsatellite markers for genetic analysis and plant breeding with emphasis on bread wheat. Euphytica.2000; 113: 163-85.

https://doi.org/10.1023/A:1003910819967.

Haigler $\mathrm{CH}$, Ivanova-Datcheva M, Hogan PS,et al. Carbon partitioning to cellulose synthesis. Plant Molecular Biology.2001; 47: 29-51.

Han ZG, Wang CB, Song XL, et al.Characteristics, development and mapping of Gossypium hirsutumderived EST-SSRs in allotetraploid cotton. Theoretical \& Applied Genetics.2006; 112: 4309. https://doi.org/10.1007/s00122-005-0142-9.

Han ZG, Guo WZ, Song XL, et al. Genetic mapping of EST-derived microsatellites from the diploid Gossypium arboreum in allotetraploid cotton. Mol Genet Genomics.2004; 272: 308-27.

https://doi.org/10.1007/s00438-004-1059-8.

Hawkins JS, Kim HR, Nason JD, et al. Differential lineage-specific amplification of transposable elements is responsible for genome size variation in Gossypium. Genome Res. 2006;16:1252-61. https://doi.org/10.1101/gr.5282906.

He XC, Qin YM, Xu Y, et al. Molecular cloning, expression profiling, and yeast complementation of 
19 beta-tubulin cDNAs from developing cotton ovules. Journal of Experimental Botany.

2008;59(10):2687-2695. https://doi.org/10.1093/jxb/ern127.

Hovav R, Udall JA, Chaudhary B, et al. The evolution of spinnable cotton fiber entailed prolonged development and a novel metabolism. PLoS Genet. 2008;4(2):e25.

https://doi.org/10.1371/journal.pgen.0040025.

Kantartzi SK, Ulloa M, Sacks E, et al. Assessing genetic diversity in Gossypium arboreum L. cultivars using genomic and EST-derived microsatellites. Genetica.2009; 136: 141-7. https://doi.org/10.1007/s10709-008-9327-x.

Kirill R, Wonhee J, Schuler GD. A web server for performing electronic PCR. Nucleic Acids Research. 2004;32:108-12. https://doi.org/10.1093/nar/gkh450.

Liu DQ, Guo XP, Lin ZX, et al. Genetic diversity of Asian Cotton (Gossypium arboreum L.) in China evaluated by microsatellite analysis. Genetic Resources \& Crop Evolution.2006; 53: 1145-52. https://doi.org/10.1007/s10722-005-1304-y.

Liu J, Qu JT, Yang C, et al. Development of genome-wide insertion and deletion markers for maize, based on next-generation sequencing data. BMC Genomics.2015; 16: 601.

https://doi.org/10.1186/s12864-015-1797-5.

Liu X, Zhao B, Zheng HJ, et al. Gossypium barbadense genome sequence provides insight into the evolution of extra-long staple fiber and specialized metabolites. Scientific Reports.2015;5: 14139. https://doi.org/10.1038/srep14139.

Tani N, Takahashi T, Iwata H, et al. A consensus linkage map for sugi (Cryptomeria japonica) from two pedigrees, based on microsatellites and expressed sequence tags. Genetics. 2003;165:1551-68.

Nazareno A, Reis M. The same but different: Monomorphic microsatellite markers as a new tool for genetic analysis. American Journal of Botany.2011; 98: 265-7.https://doi.org/10.3732/ajb.1100163.

Nguyen TB, Giband M, Brottier P, et al. 2004. Wide coverage of the tetraploid cotton genome using newly developed microsatellite markers. Theoretical \& Applied Genetics.2014; 109: 16775. https://doi.org/10.1007/s00122-004-1612-1. 
Paterson AH, Brubaker CL, Wendel JF. A rapid method for extraction of cotton ( Gossypium spp.)

genomic DNA suitable for RFLP or PCR analysis. Plant molecular biology reporter.1993; 11: 122-

7. https://doi.org/10.1007/BF02670470.

Powell W, Machray GC, Provan J. Polymorphism revealed by simple sequence repeats. Trends Plant

Sci.1996; 1: 215-22. https://doi.org/10.1016/1360-1385(96)86898-1.

Qayyum A, Murtaza N, Azhar F, et al. Biodiversity and nature of gene action for oil and protein contents in Gossypium hirsutum L. estimated by SSR markers. Journal of Food Agriculture and Environment. 2009; 7: 590-3.

Reddy, OUK, Pepper AE, Abdurakhmonov I, et al. New dinucleotide and trinucleotide microsatellite marker resources for cotton genome research. Journal of Cotton Science. 2001; 5: 103-13.

Rohlf FJ. NTSYS-pc: Microcomputer Programs for Numerical Taxonomy and Multivariate Analysis. The American Statistician. 1987; 41 (4): 330. DOI:10.2307/2684761.

Rozen S, Skaletsky H. Primer3 on the WWW for general users and for biologist programmers. Methods

in Molecular Biology. 2000;132: 365. https://doi.org/10.1385/1-59259-192-2:365.

Ruan YL. Rapid cell expansion and cellulose synthesis regulated by plasmodesmata and sugar: insights from the single-celled cotton fibre. Functional Plant Biology. 2007; 34: 1-10.

DOI: 10.1071/FP06234.

Santos FR, Pena SDJ, Epplen JT. Genetic and population study of a Y-linked tetranucleotide repeat

DNA polymorphism with a simple non-isotopic technique. Hum Genet.1993; 90: 655-

56. https://doi.org/10.1007/BF00202486.

Shi JQ, Huang SM, Zhan JP, et al. Genome-wide microsatellite characterization and marker development in the sequenced Brassica crop species. Dna Research. 2014; 21: 53-

68. https://doi.org/10.1093/dnares/dst040.

Thiel T, Michalek W, Varshney R, et al. Exploiting EST databases for the development and characterization of gene-derived SSR-markers in barley (Hordeum vulgare L.). Theoretical \& Applied Genetics. 2003; 106: 411-22. https://doi.org/10.1007/s00122-002-1031-0.

Wang Q, Fang L, Chen J, et al. Genome-wide mining, characterization, and development of microsatellite markers in Gossypium species. Scientific Reports. 2015; 5: 10638. https://doi.org/10.1038/srep10638.

Xie H, Sui Y, Chang FQ, et al. SSR allelic variation in almond (Prunus dulcis Mill.). Theoretical and 
Applied Genetics. 2006; 112: 366-72. https://doi.org/10.1007/s00122-005-0138-5.

Yang SS, Cheung F, Lee JJ, et al. Accumulation of genome-specific transcripts, transcription factors and phytohormonal regulators during early stages of fiber cell development in allotetraploid cotton.

The Plant Journal. 2006; 47: 761-75. https://doi.org/10.1111/j.1365-313X.2006.02829.x

Yves AG, Stephane B, Tony A, et al. Transcript profiling during fiber development identifies pathways in secondary metabolism and cell wall structure that may contribute to cotton fiber quality. Plant \& Cell Physiology. 2009; 50: 1364-81._https://doi.org/10.1093/pcp/pcp084.

Zhang HB, Li YN, Wang BH, et al. Recent advances in cotton genomics. International Journal of Plant Genomics. 2008; 2008: 742304._https://doi.org/10.1155/2008/742304.

Zhang JF, Yang L, Cantrell RG, et al. Molecular marker diversity and field performance in commercial cotton cultivars evaluated in the Southwestern USA. Crop Science. 2005; 45: 148390. https://doi.org/10.2135/cropsci2004.0581.

Zhang M, Zheng XL, Song SQ, et al. Spatiotemporal manipulation of auxin biosynthesis in cotton ovule epidermal cells enhances fiber yield and quality. Nature Biotechnology.2011; 29: 453-8.

https://doi.org/10.1038/nbt.1843.

Zhang PP, Wang XQ, Yang Y, et al. Isolation, characterization, and mapping of genomic microsatellite markers for the first time in Sea-Island Cotton (Gossypium barbadense). Acta Agronomic Sinica.2009; 35: 1013-20. DOI:10.3724/SP.J.1006.2009.01013.

Zhou Q, Lou D, Ma LC,et al. Development and cross-species transferability of EST-SSR markers in Siberian wildrye (Elymus sibiricus L.) using Illumina sequencing. Scientific Reports.2016; 6:

20549. https://doi.org/10.1038/srep20549.

Zou CS, Lu CR, Zhang YP, et al. Distribution and characterization of simple sequence repeats in Gossypium raimondii genome. Bioinformation.2012; 8: 801-6.

https://doi.org/10.6026/97320630008801.

\section{Figures}




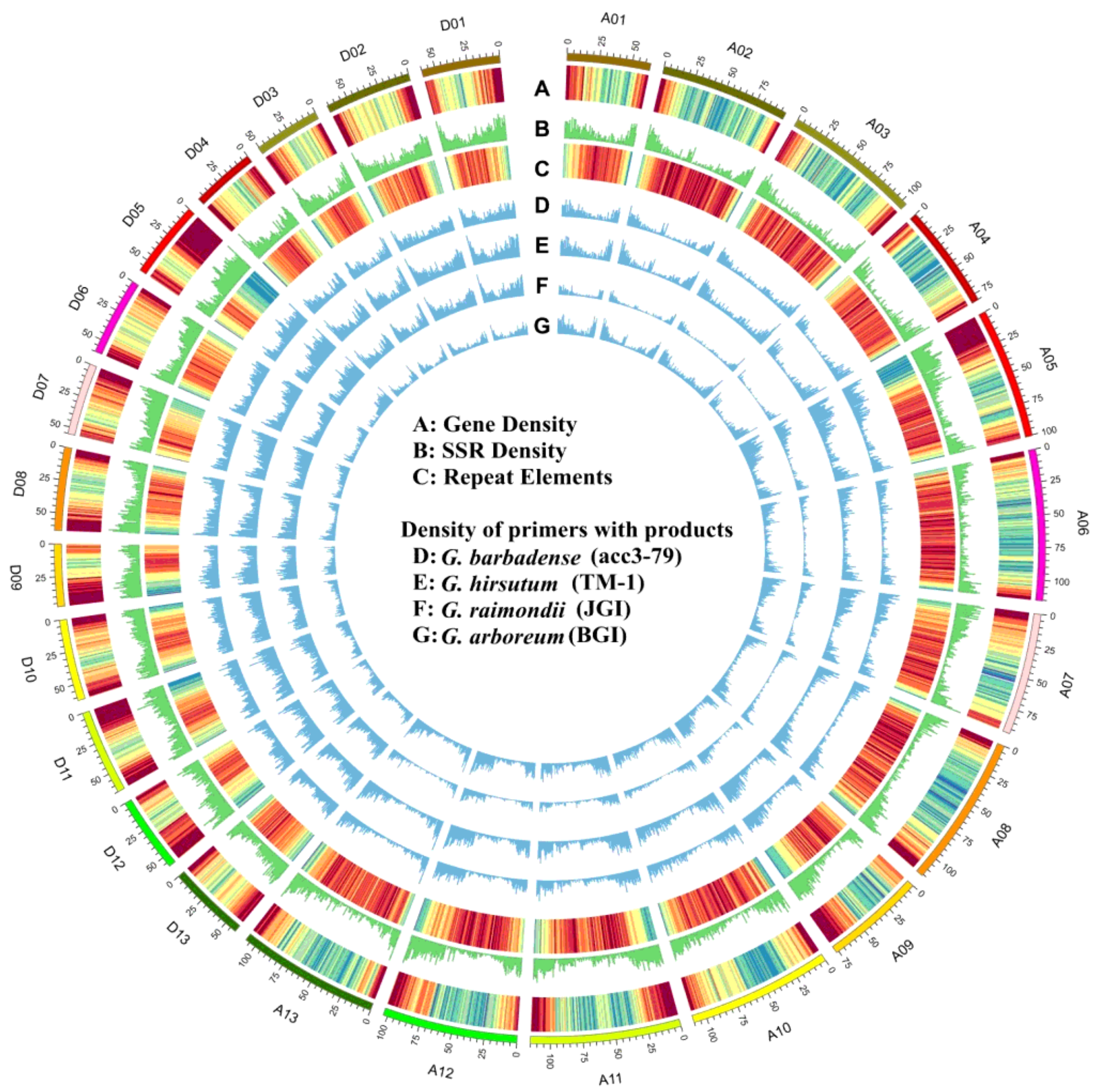

Figure 1

Heat map of gene density, SSR density and repeat elements of G. barbadense cv. Xinhai21 and density of primer sets with products. (A, C) Colors from red to yellow, to green indicate density from high to low (B, $D-G)$. The higher the peak is, the higher the density is. 

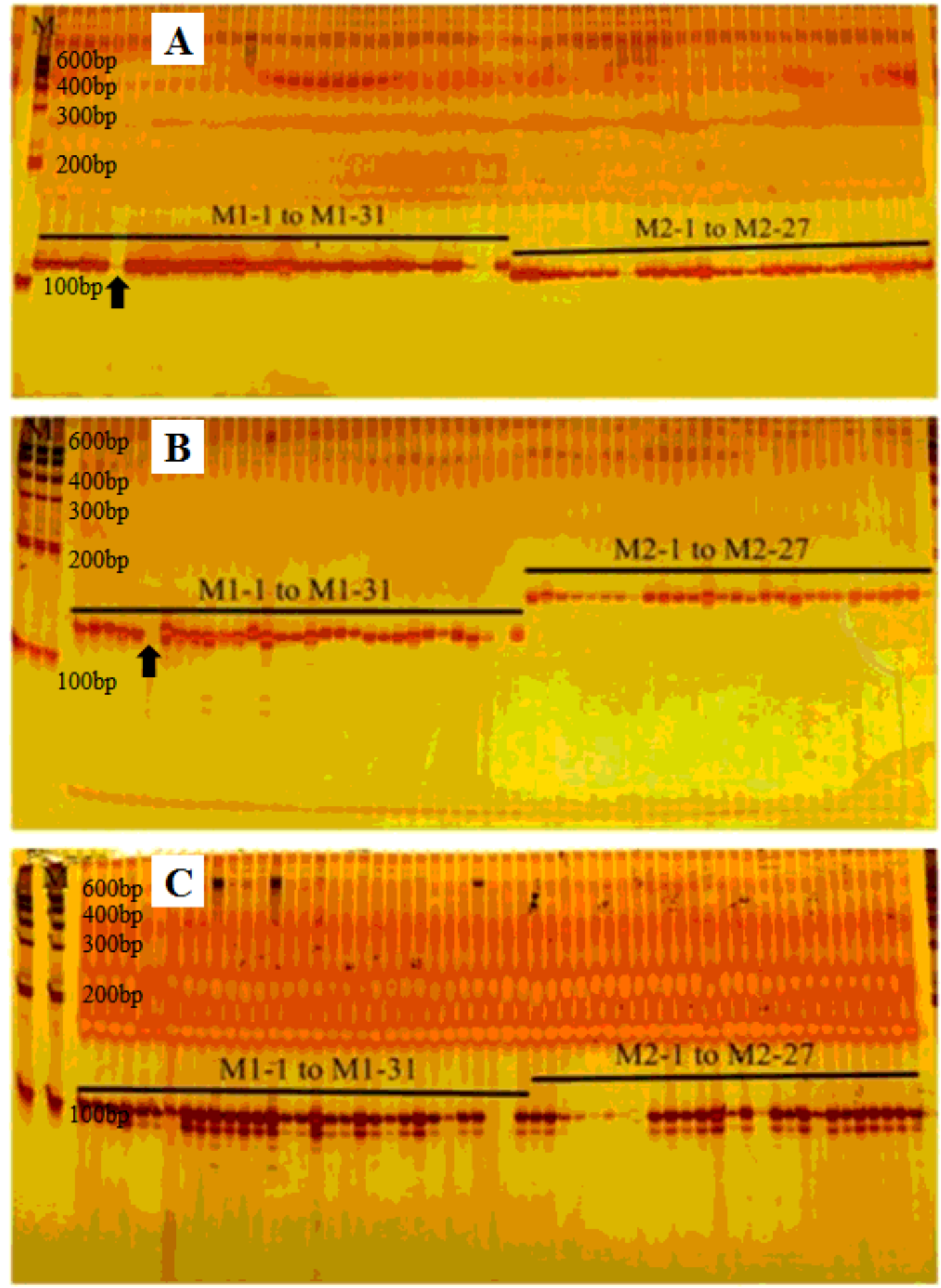

Figure 2

Gel electrophoresis profiles of SSR marker primer sets. (A) Primer XHSSR8, showing length polymorphism (B) Primer XHSSR32, showing length polymorphism (C) Primer XHSSR42, no polymorphism. DNA molecular standard with length (bp) is indicated on the left. Genotypes of $30 \mathrm{G}$. hirsutum and $27 \mathrm{G}$. barbadense are indicated in lanes M1-1 to M1-30 and M2-1 to M2-27, respectively. The lanes are pointed with black arrows indicate that no samples were loaded. 


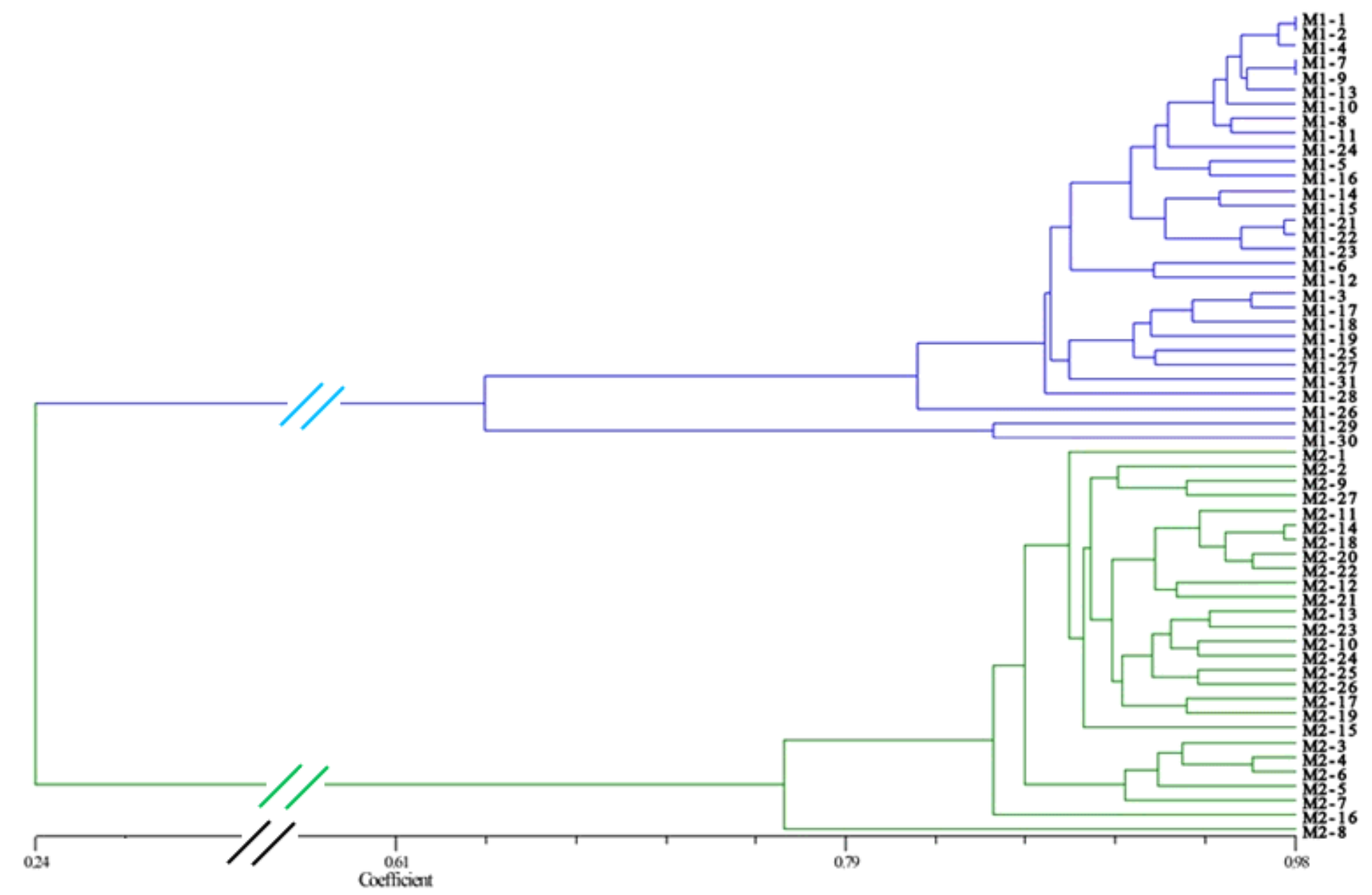

Figure 3

Phylogenetic relationship among $30 \mathrm{G}$. hirsutum and $27 \mathrm{G}$. barbadense accessions. At a similarity coefficient $\geq 0.61, G$. hirsutum and $G$. barbadense accessions were divided into two subgroups.

\section{Supplementary Files}

This is a list of supplementary files associated with this preprint. Click to download.

- TableS1.Repeatsequencesof476typesofmotifs.xlsx

- TableS2.Frequencyofdifferentmotifsinthegenome.xlsx

- TableS3.InformationofSSRmarkerprimersets.xlsx

- TableS4.Oneproductprimersetsinsilicoanalysis.xIsx

- TableS5.GOandKEGGenrichmentforXinhai21genes.xlsx

- TableS6.GenescontainingSSRmarkers.xlsx

- TableS7.300SSRprimersforgeneticdiversityanalysis.xlsx

- TableS8.Detailedinformationofthe57accessions.xlsx 